\title{
Transcriptional profiling of skeletal muscle reveals starvation response and compensatory growth in Spinibarbus hollandi
}

Yang Yang, Huiqiang Zhou, Liping Hou, Ke Xing ${ }^{*}$ and Hu Shu*

\begin{abstract}
Background: Spinibarbus hollandi is an economically important fish species in southern China. This fish is known to have nutritional and medicinal properties; however, its farming is limited by its slow growth rate. In the present study, we observed that a compensatory growth phenomenon could be induced by adequate refeeding following 7 days of fasting in S. hollandi. To understand the starvation response and compensatory growth mechanisms in this fish, the muscle transcriptomes of $S$. hollandi under control, fasting, and refeeding conditions were profiled using next-generation sequencing (NGS) techniques.

Results: More than $4.45 \times 10^{8}$ quality-filtered 150-base-pair Illumina reads were obtained from all nine muscle samples. De novo assemblies yielded a total of 156,735 unigenes, among which 142,918 (91.18\%) could be annotated in at least one available database. After 7 days of fasting, 2422 differentially expressed genes were detected, including 1510 up-regulated genes and 912 down-regulated genes. Genes involved in fat, protein, and carbohydrate metabolism were significantly up-regulated, and genes associated with the cell cycle, DNA replication, and immune and cellular structures were inhibited during fasting. After refeeding, 84 up-regulated genes and 16 down-regulated genes were identified. Many genes encoding the components of myofibers were significantly upregulated. Histological analysis of muscle verified the important role of muscle hypertrophy in compensatory growth.

Conclusion: In the present work, we reported the transcriptome profiles of S. hollandi muscle under different conditions. During fasting, the genes involved in the mobilization of stored energy were up-regulated, while the genes associated with growth were down-regulated. After refeeding, muscle hypertrophy contributed to the recovery of growth. The results of this study may help to elucidate the mechanisms underlying the starvation response and compensatory growth.
\end{abstract}

Keywords: Spinibarbus hollandi, Starvation response, Compensatory growth, Muscle

\section{Background}

Unfavorable environmental conditions, such as extreme temperature, depression, and hypoxic conditions, and human activities, such as transportation, can inhibit feeding behavior in fish, resulting in the starvation of fish in aquaculture. Starvation can influence growth, development, reproduction, and immunity in animals [1, 2]. Organisms usually maintain metabolic homeostasis

\footnotetext{
* Correspondence: xingk@mail.sysu.edu.cn; shuhu001@126.com

School of Life Science, Guangzhou University, Guangzhou 510006, China
}

during starvation by changing their behavior and activating various physiological and biochemical adaptive mechanisms. Recent studies have shown that lipid metabolism could be increased, and energy reserves could be mobilized, to cope with starvation in Piaractus mesopotamicus [3]. In rainbow trout (Oncorhynchus mykiss), it was found that genes related to protein degradation and amino acid metabolism increased, while genes involved in protein synthesis decreased under starvation [4]. In yellow croaker (Larimichthys crocea), a number of

(C) The Author(s). 2019 Open Access This article is distributed under the terms of the Creative Commons Attribution 4.0 International License (http://creativecommons.org/licenses/by/4.0/), which permits unrestricted use, distribution, and 
genes associated with carbohydrate metabolism also increased during fasting conditions [5]. In some fish species, food shortages have been shown to weaken immunity and inhibit body growth because of decreased energy consumption [6,7].

Previous studies have shown that some fish have the ability to tolerate short-term food deprivation or fasting. Animals fed to satiation following continuous fasting have the potential to exhibit increased feeding intensity compared with continuously fed controls, resulting in the acceleration of growth; this phenomenon is defined as compensatory growth [8], which is known to occur in a wide range of fish species, such as Atlantic salmon (Salmo salar) [9], barramundi (Lates calcarifer) [10], channel catfish (Ictalurus punctatus) [11], tongue sole (Cynoglossus semilaevis) [12], European minnow (Phoxinus phoxinus) [13], and gibel carp (Carassius auratus gibelio) [14]. However, the regulatory mechanisms governing this phenomenon have not been thoroughly elucidated in fish.

Musculature provides the largest store of protein in the body of fish; muscle proteins are the most important energy source and are preferentially mobilized in vital organs during long-term food deprivation [15]. Furthermore, the muscle is the main edible tissue of most fish species and accounts for at least $50 \%$ of the body weight in most commercial fish species [16]. Previous studies have shown that muscle may play an important role in maintaining normal metabolism during dietary restriction due to an increase in protein degradation of the white muscle during starvation [15]. The investigation of the morphology and molecular changes in muscle during food deprivation can facilitate a better understanding of the starvation response and compensatory growth mechanisms.

Spinibarbus hollandi (also called army fish) is an endemic Cyprinidae species in southeastern China and is primarily distributed in Guangdong, Guangxi, Hunan, Hubei, Fujian, and Anhui Provinces. This fish is omnivorous, is easy to rear, and has already received increasing attention owing to its high nutritional and medicinal value [17]. However, the low growth rate seriously restricts the production efficiency and popularization of $S$. hollandi [17]. In addition, this fish species is sensitive to external stimuli, environmental changes and artificial stimulation, which can easily result in fasting for several days. In our previous work, we attempted to enhance the growth rate of $S$. hollandi by improving the rearing conditions and crossbreeding. The molecular markers associated with growth traits were developed in S. hollandi $[17,18]$. However, studies on the effects of fasting and refeeding on the growing status of this species are deficient. The underlying physiological responses and gene expression changes associated with growth depression due to feed restriction have not been elucidated.
In the present study, the growth compensation phenomenon was observed in S. hollandi. To understand the regulatory mechanisms underlying the starvation response and growth compensation, comparative transcriptome analyses on skeletal muscle of different feeding statuses were performed in S. hollandi. The results showed that muscle overgrowth is an important reason for growth compensation. Therefore, a histological analysis of muscle was carried out to verify the conclusions obtained from transcriptome data.

\section{Results}

As shown in Fig. 1a, a total of 210 mixed-sex, full-sib $S$. hollandi were randomly divided into a test group and a control group. For the test group, fish were first deprived of food for 7 days and then fed twice a day to apparent satiation for 33 days. For the control group, fish were fed twice a day continuously for 40 days (Fig. 1a). Both groups were sampled sequentially on days $0,7,14$ and 40 , and the growth traits, including weight, body length, total length, thickness and height, were measured. The muscle tissue transcriptomes were profiled by RNAsequencing (RNA-seq) at day 7 for the control group and test group (after 7 days of fasting) and 14 for the test group (after 7 days of refeeding).

\section{Compensatory growth after fasting-refeeding treatment}

After fasting, with the exception of body thickness, the body weight, total length, body length, and body height were decreased and significantly lower than those of the control group ( $p<0.05$ Student's t-test; Fig. 1). After 7 days of refeeding, no remarkable difference could be detected between the test group and the control. Finally, at the end of the experiment, all growth traits were recovered (Fig. 1). Taken together, these results suggest effective growth compensation in S. hollandi after fasting-refeeding treatment. The daily gains of growth traits can be found in Additional file 1: Figure S1.

\section{Sequencing data and de novo assembly}

The variation among individuals was minimized by mixing equal amounts of RNA from five samples in the same group. For each group, three replicated mixed RNA pools were generated and used for cDNA library preparation and RNA-seq thereafter. A total of $4.63 \times$ $10^{8}$ raw reads $(150 \mathrm{bp})$ were obtained from nine cDNA libraries. After removing the low-quality sequences, approximately $4.45 \times 10^{8}$ clean reads with $66.71 \mathrm{~Gb}$ sequences were generated. Then, 355,268 transcripts (ranging from 201 to 27,096 bp) were generated through de novo assembly. The longest transcript of a gene was regarded as a unigene for further analyses. A total of 156,735 unigenes with $103.5 \mathrm{Mb}$ sequences were 

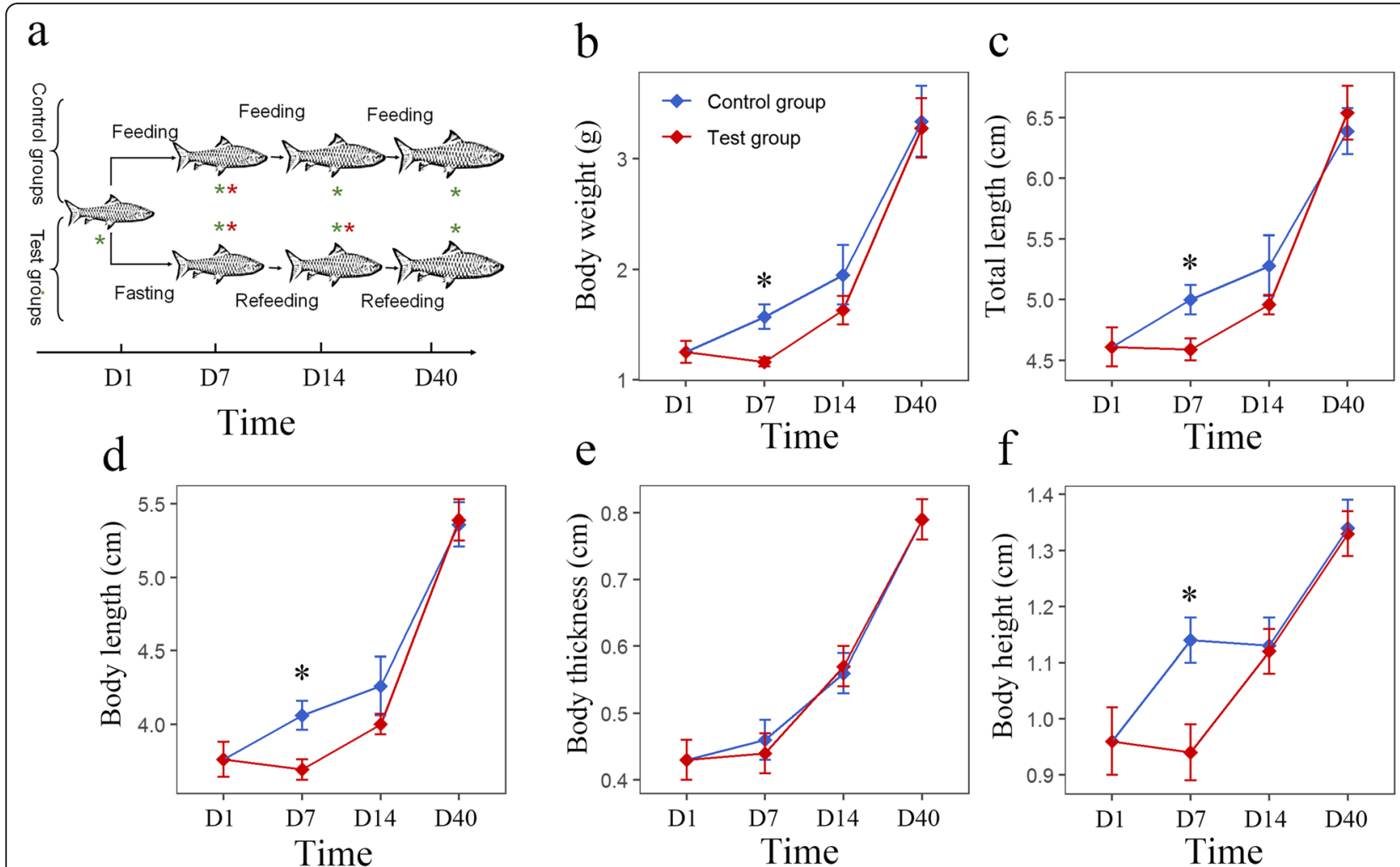

Fig. 1 Experimental procedure, growth curves. a Illustration of the experiment and sample processes performed in this study. Red asterisks indicate that groups were used for RNA-seq; green asterisks indicate that groups were used for qRT-PCR; the horizontal axis indicates the time of experiment. b-f Growth curve of body weight, total length, body length, body thickness and body height, respectively. Asterisks indicate significant differences between the control and test groups ( ${ }^{*} p<0.05$; Student's $t$-test)

obtained with an average length of $660 \mathrm{bp}$. The N50 and N90 lengths were 1061 and $224 \mathrm{bp}$, respectively.

\section{Annotation and functional analysis of muscle unigenes}

Among all unigenes, 142,918 (91.18\%) could be annotated in at least one database of non-redundant protein $(\mathrm{Nr})$, non-redundant nucleotide $(\mathrm{Nt}) \mathrm{NCBI}$ database, Pfam, KOG, Swiss-Prot, KEGG, and GO, with 10,438 $(6.65 \%)$ unigenes annotated in all these databases. Approximately $54,408(34.71 \%)$ unigenes were annotated in the $\mathrm{Nr}$ database, of which nearly $66.5 \%$ had the highest similarity with Danio rerio followed by Astyanax mexicanus (5.8\%), Clupea harengus (2.5\%), Larimichthys crocea (1.8\%), and Oncorhynchus mykiss (1.7\%).

The functional prediction and classification of all unigenes were performed against the GO database. A total of 42,174 (26.91\%) unigenes were classified into 55 GO terms, including 10 molecular function, 20 cellular components, and 25 biological process terms. Cellular process, binding, metabolic process, single-organism process, and catalytic activity were the five most abundant functional terms at the second GO level (Fig. 2a). Furthermore, the assembled unigenes were aligned to the COG database for phylogenetic classification. A total of 16,978 (10.83\%) unigenes were divided into 26 functional categories (Fig. 2b); the largest category was signal transduction mechanisms with 3198 unigenes followed by general function prediction only (2495); posttranslational modification, protein turnover, chaperones (1773); and cytoskeleton (1343). The biological pathways were analyzed by annotating all assembled unigenes against the KEGG database, and 29,841 (19.04\%) unigenes were matched in five categories, including organismal systems $(11,222 ; 37.61 \%)$, environmental information processing (7138; 23.92\%), cellular processes $(5912 ; 19.81 \%)$, metabolism $(5089 ; 17.05 \%)$, and genetic information processing (3521; 11.80\%). These categories contained 232 pathways with 1 to 1147 unigenes that were involved in an individual pathway. Among all KEGG pathways, the PI3K-Akt signaling pathway, focal adhesion, regulation of actin cytoskeleton, endocytosis, and MAPK signaling pathway were significantly enriched with more than 800 unigenes (Fig. 2c).

\section{Identification of DEGs in different feeding conditions}

A total of 4503 unigenes showed significant differential expression among the control, fasting and refeeding groups. Of these differentially expressed 


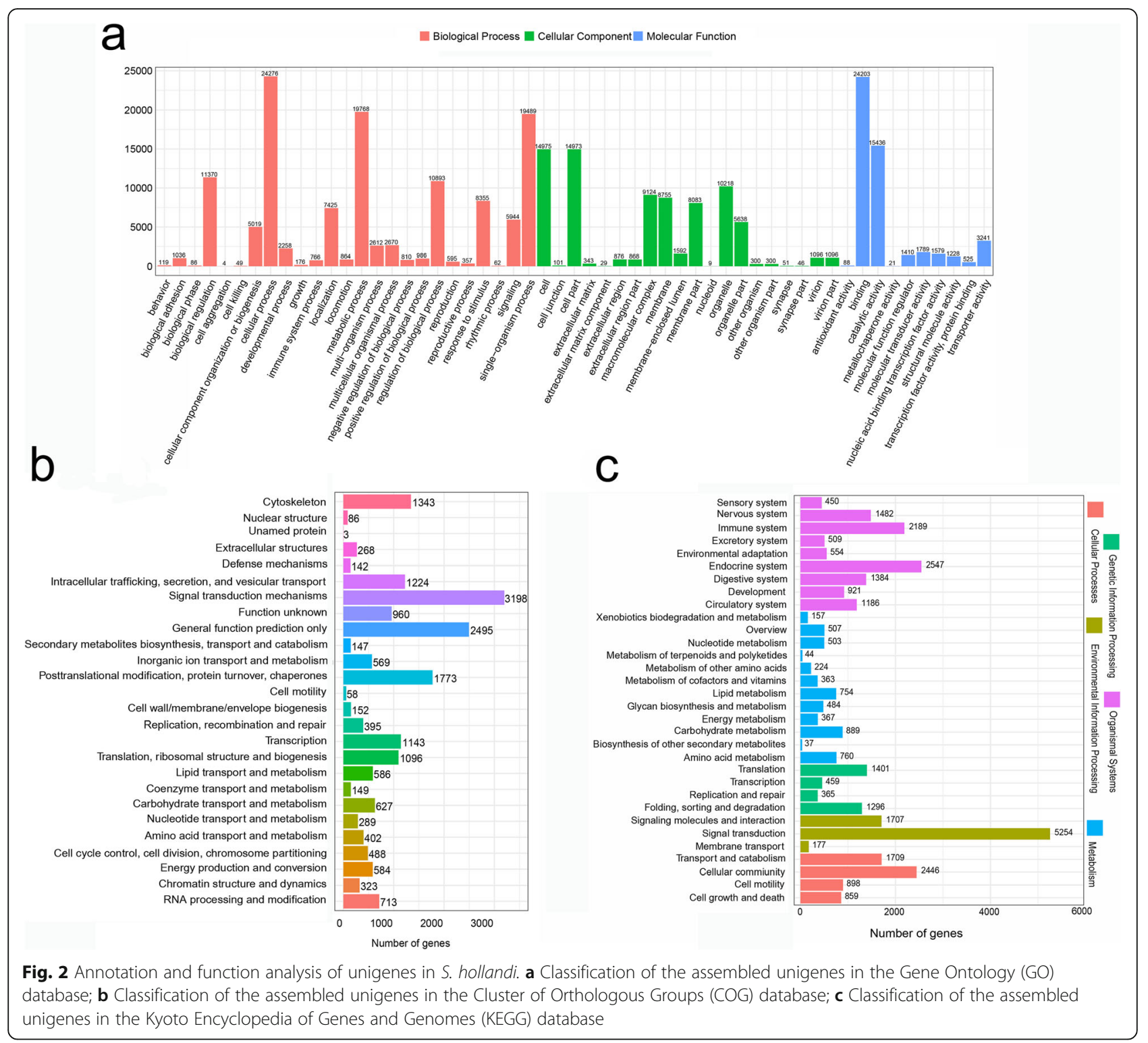

genes (DEGs), 2422 were found between the control and fasting groups, 110 were found between the control and refeeding groups, and 3970 were found between the fasting and refeeding groups (Fig. 3a, b and Additional file 2: Table S2). The overall expression profiles of control group and refeeding group were similar $\left(R^{2}=0.95, p<2.2 \mathrm{e}-16\right.$; only the 4503 DEGs were considered), and the DEGs identified from fasting compared to control comparison and fasting compared refeeding comparison were largely overlapped (Fig. 3a, b). We mainly focused on genes significantly changed in fasting group compared to control and genes significantly changed in refeeding group compared to control in our following analysis.

\section{Analysis of DEGs after fasting in S. hollandi}

After 7 days of fasting, we detected 2422 DEGs, which included 1510 up-regulated and 912 down-regulated genes (Additional file 2: Table S2). Approximately 331 up-regulated DEGs mapped to 284 KEGG pathways, 26 of which were significantly enriched (corrected $p$-value $<$ 0.05; Fig. 4a). The top five significantly enriched pathways were the AMPK signaling pathway (ko04152), insulin resistance (ko04931), insulin signaling pathway (ko04910), fatty acid degradation (ko00071), and adipocytokine signaling pathway (ko04920). Many metabolism-relevant genes were significantly upregulated in the muscle after 7 days of fasting (Fig. $4 \mathrm{~b}$ ). The genes associated with lipid metabolism (PNPLA2, 

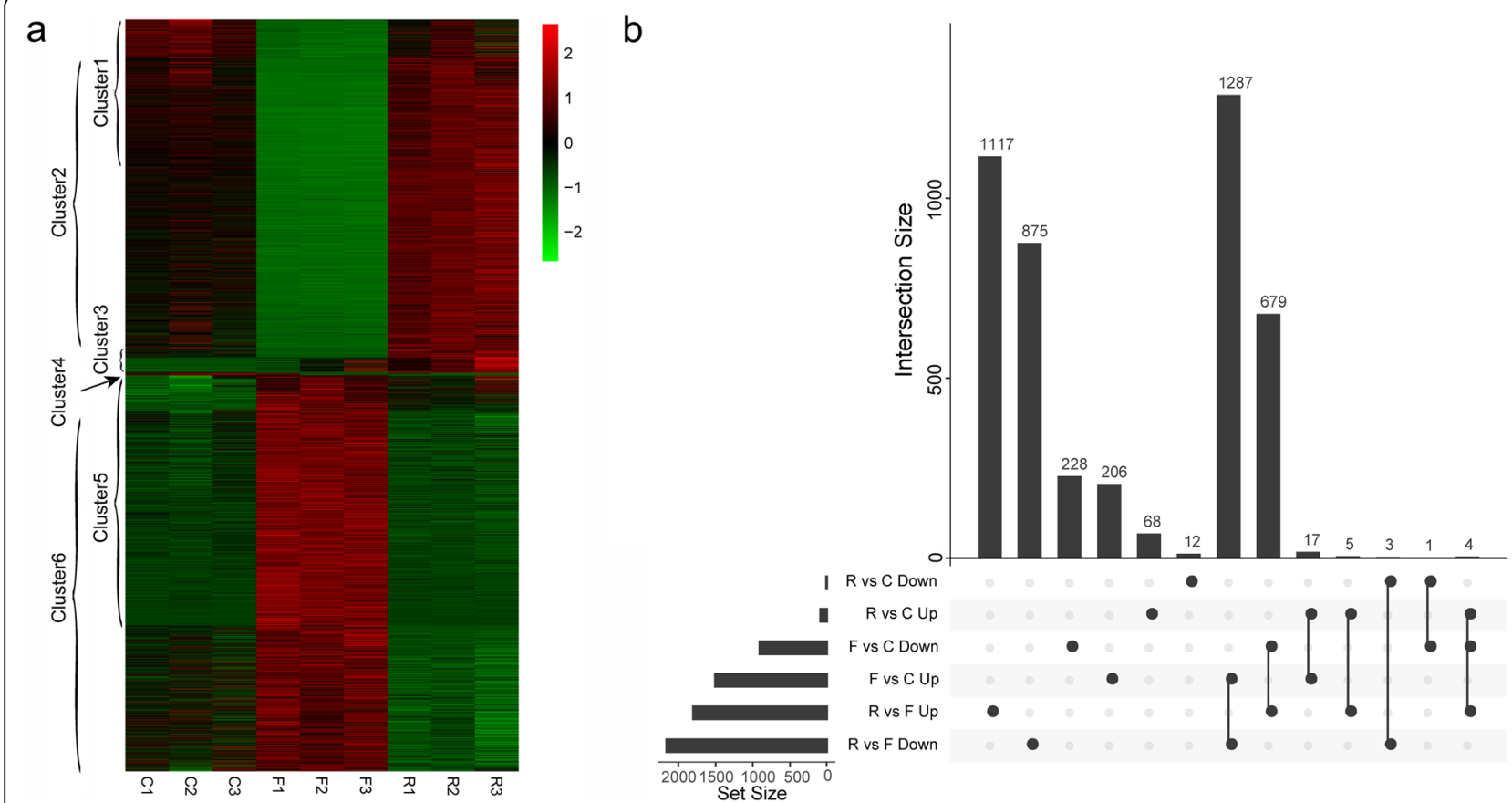

Fig. 3 Overall transcriptome profiles of all DEGs. a Heatmap of all DEGs. Green and red indicate the low and high expression levels, respectively. C1, C2 and C3 indicate the control group, F1, F2 and F3 indicate the fasting group, and R1, R2 and R3 indicate the refeeding group. Cluster1 contains genes significantly down regulated in fasting group compared to control, with the exception of genes also significantly changed in refeeding group compared to control; Cluster2 contains genes significantly down regulated in fasting group compared to refeeding group, with the exception of genes also significantly changed in refeeding group compared to control; Cluster3 contains genes significantly up regulated in refeeding group compared to control; Cluster4 contains genes significantly down regulated in refeeding group compared to control; Cluster 5 contains genes significantly up regulated in fasting group compared to control, with the exception of genes also significantly changed in refeeding group compared to control; Cluster6 contains genes significantly up regulated in fasting group compared to refeeding group, with the exception of genes significantly changed in refeeding group compared to control. The full list of these DEGs can be found in Additional file 2: Table S2. $\mathbf{b}$ UpSet chart showing intersection between different DEG sets from various comparisons. The vertical bar plot reports the intersection size, the dot plot reports the set participation in the intersection, and the horizontal bar plot reports the set sizes

SLC27A1, SLC27A3, HADH, HADHA, LPIN1, ACACB1, $A C A C B 2, C P T 1 A$, and $C P T 1 B)$, protein degradation and metabolism (USP19, RNF25, USP25, USP28, UBE2A, $P S M B 7$, and PSMG3), and glycometabolism (PPP1CB, PFKFB1, GSK3B, and GYS) were significantly upregulated. In addition, some genes associated with signal transduction (FOXO3, FOXO4, PIK3, and ARS1) were up-regulated during fasting. To validate the results from RNA-seq, the expression profiles of RNF25 and SLC27A3 were analyzed by using quantitative PCR (qPCR). The results were similar to the RNA-seq results, in which the expression of these two genes was significantly increased after 7 days of fasting and then returned to normal levels after refeeding (Fig. 4c).

A total of 316 down-regulated DEGs were mapped to 243 pathways in the KEGG database, of which 26 pathways (Fig. 5a) were significantly enriched (corrected $p$-value $<0.05)$. The top five significantly enriched pathways were cell cycle (ko04110), protein digestion and absorption (ko04974), ECM-receptor interaction (ko04512), PI3K-Akt signaling pathway (ko04151), and phagosome (ko04145). The expression profiles of some of the down-regulated DEGs from RNA-seq are shown in Fig. 5b. Genes associated with the cell cycle (HRAS, CDK1, CDK2, CDC6, FGF6, CCNA2, Wee1, and CCNB1), DNA replication (EIF4E1A, PCNA, POLD1, MCM1, MCM2, MCM3, MCM4, MCM5, and MCM6), extracellular matrix (ITGA4, COL1A, LAMC1, FN1, TN, and DAG1), protein synthesis (RPN2, GCS1, HSPA5, PDIA3, PDIA4, $L M A N 1$, and ERLEC1), and immune and stress tolerance (ARHGEF2, TUBA, TUBB, FYN, MHCII, TAP1, HSP70, HSP90, and CTSB) were significantly downregulated. The expression of CDK1 and MHCII in the control and test groups was analyzed using qRT-PCR (Fig. 5c). The expression of CDK1 was significantly down-regulated after 7 days of fasting, and then returned to normal levels after refeeding. MHCII was significantly down-regulated after 7 days of fasting, and its expression gradually increased. 


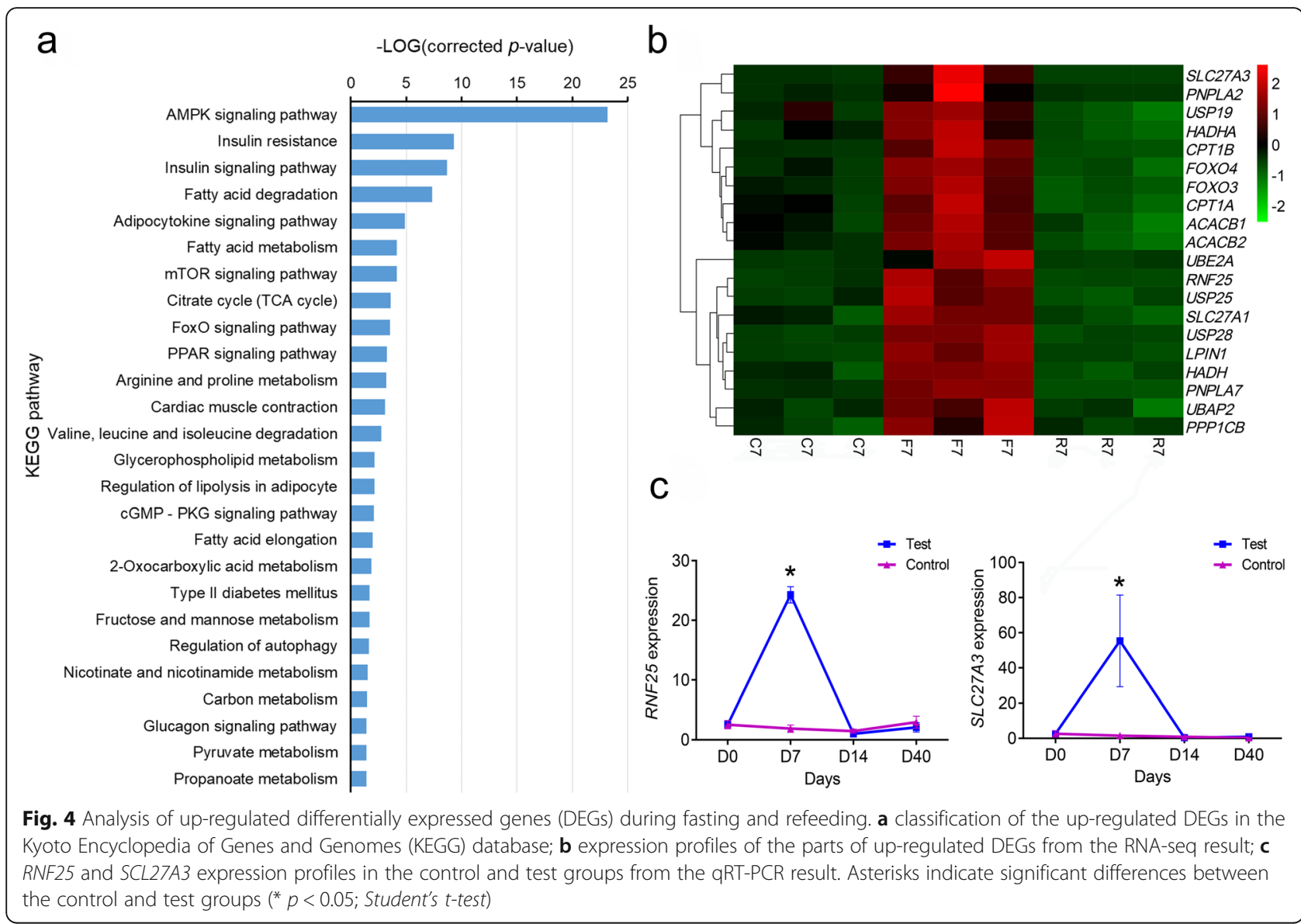

\section{Analysis of DEGs after refeeding}

After refeeding, we detected a total of 3970 DEGs between the fasting and refeeding groups, of which 1805 were up-regulated and 2165 were down-regulated (Additional file 2: Table S2). Compared with the control group, a total of 110 genes were significantly changed, including 94 DEGs and 16 DEGs that were up-regulated and down-regulated. It's worth noting that among the 94 up regulated genes in the refeeding group, 38 of them increased in one or two fasting samples too. These genes, thus, may not related to the compensatory growth. In the remaining DEGs, 9 genes involved in myofiber structure (ACTC1, ACT2, ACTA1, MHC, MLC1, MYL2, TM1, TNT, and TNNT3) were significantly up-regulated (Additional file 1: Figure S2 and Table 1). In addition, genes involved in collagen (COL3A1, COL1A2, and COL5A2), ribosomal protein (RPL22L1), protein synthesis (INFB), and energy metabolism (ATPD, GAPDH, ND2, and PDHB) were also upregulated (Table 1 ).

Three genes, ACT2, ACTA1, and TNNT3, were used for expression trend analysis between the control and test groups. The ACT2 expression significantly decreased after fasting for 7 days $(p=0.013$; Student's $t$-test, $d f=4)$ compared with the control group at the same point and then sharply increased and became significantly higher than that in the control group with refeeding for 7 days; after refeeding for 33 days, the ACT2 expression decreased, but it was still significantly higher than that in the control group $\left(p=1.0 \times 10^{-5}\right.$; Student's t-test, $\left.d f=4\right)$ (Fig. 6a). The expression profile of ACTA1 had the same pattern as that of $A C T 2$ : after fasting, its expression significantly decreased, whereas after refeeding, its expression significantly increased and then gradually decreased $\left(p=8.05 \times 10^{-4}\right.$; Student's $t$-test,$\left.d f=4\right)($ Fig. $6 \mathrm{~b})$. The expression change of TNNT3 was similar to ACTA1 and $A C T 2$; however, after refeeding for 33 days, no significant difference was noted in its expression between the control and refeeding groups (Fig. 6c).

\section{qRT-PCR validation of DEGs from RNA-Seq}

A total of ten DEGs of different functions were randomly selected to verify the RNA-seq results using quantitative real-time PCR (qRT-PCR), including genes involved in protein degradation (RNF25), lipid metabolism (SLC27A3), cell cycle (CDK1 and TMSB), immunity (MCHII and TAP1), DNA replication (MCM4) and myofiber structure (ACTA1, ACT2 and TNNT3). The 


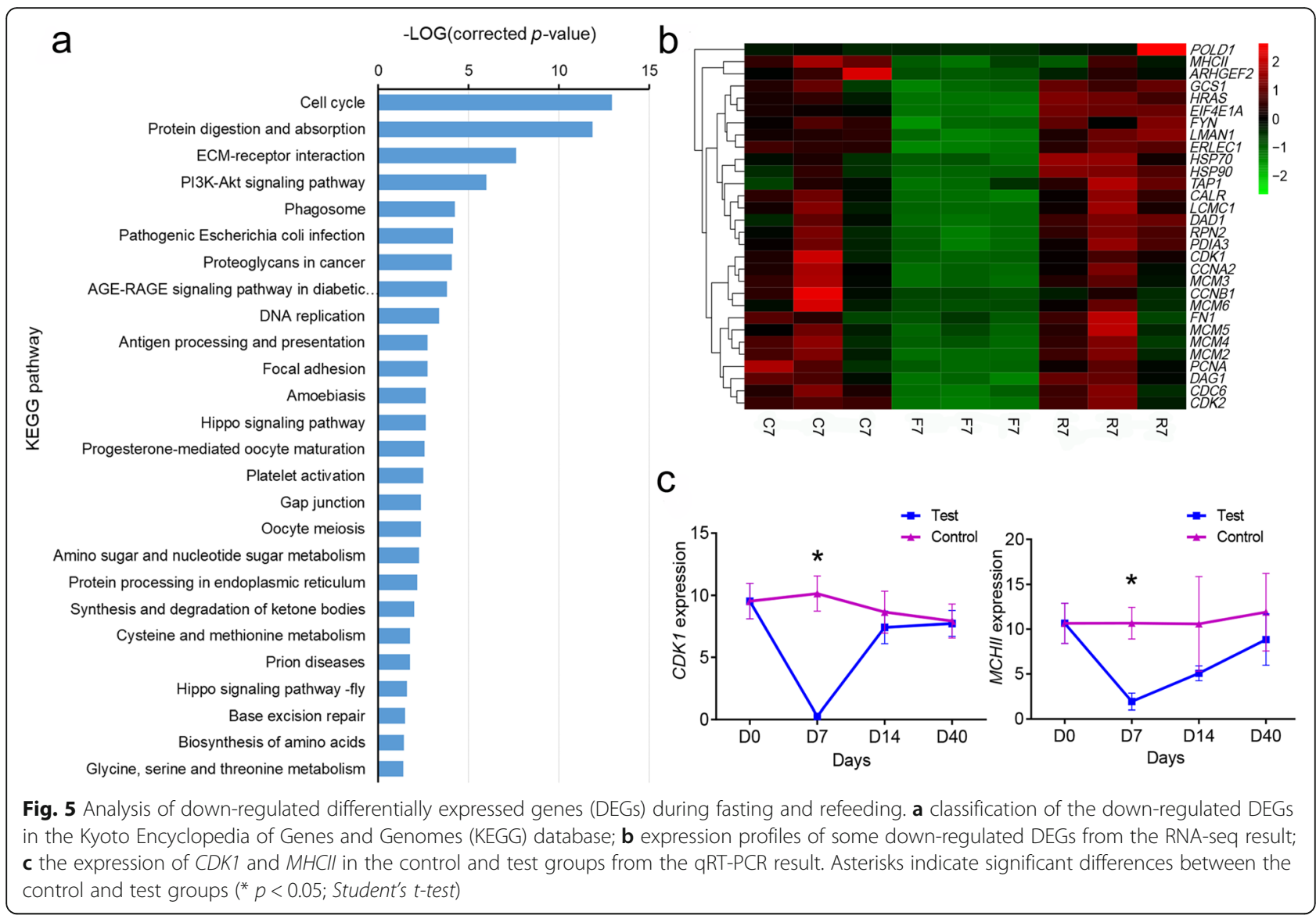

expression changes of these genes were analyzed under fasting and refeeding conditions. Under both fasting and refeeding conditions, the gene expression changes obtained from qRT-PCR were remarkably consistent with those obtained from the RNA-Seq result, with $R^{2}$ values of 0.9145 and 0.9282 (Fig. 7), respectively. The analyses confirmed the reliability and accuracy of the RNA-seq result.

\section{Changes in myofibers during fasting and refeeding periods}

The dimensions of myofibers were analyzed using histology. The myofiber sections of the control groups are shown in Fig. 8a-c and those of the test groups are shown in Fig. $8 \mathrm{~d}-\mathrm{f}$. The shortest and longest diameters of myofibers were measured (Fig. 8g, h). After 7 days, the shortest and longest diameters of the test group were $32.86 \pm 0.98$ and $36.30 \pm 1.07 \mu \mathrm{m}$, respectively, which were significantly shorter than those of the control group (37.80 \pm 1.96 and $42.14 \pm 2.09 \mu \mathrm{m}, p=0.014$ and $p=0.026$; Student's $t$-test). After 7 days of refeeding, the mean longest diameter of the test group of the refeeding group was still significantly shorter than that of the control group ( $p=0.043$; Student's $t$-test); however, no significant difference was noted between their shortest diameters $(p=0.203$; Student's $t$-test $)$. In addition, at the end of 40 days, no significant differences were noted in both diameters $(p=0.942$ and $p=0.924$, Student's t-test $)$.

\section{Discussion}

In this study, a compensatory growth phenomenon was observed in $S$. hollandi that were refed after fasting for 7 days. During fasting, the growth of S. hollandi was inhibited; after 33 days of refeeding, the weight of the fasted fish almost recovered to the normal weight of the control group. In S. hollandi, starvation increased the expression of genes involved in fat, protein, and carbohydrate metabolism but reduced the expression of genes associated with the cell cycle, DNA replication, and immune and cellular structures. The compensatory growth seemed to mainly result from muscle overgrowth; many genes involved in myofiber development were significantly upregulated. Histological analyses of muscle suggested that starvation inhibited myofiber growth but that refeeding after fasting induced myofibril overgrowth. Fasting also inhibited the growth of $S$. hollandi overall; the body weight of fasted fish even decreased slightly during fasting. A reduction in body mass may be the most obvious response to starvation. 
Table 1 Up-regulated differentially expressed genes (DEGs) after refeeding

\begin{tabular}{|c|c|c|c|c|}
\hline Gene_ID & $\log 2 \mathrm{FC}$ & padj & Swissprot Description & Symbol \\
\hline TRINITY_DN53185_c1_g5 & 14.71 & $5.04 \mathrm{E}-14$ & Uncharacterized protein ORF91 & \\
\hline TRINITY_DN28038_C0_g1 & 11.91 & $2.30 \mathrm{E}-10$ & Cell division protein FtsZ & FTSZ \\
\hline TRINITY_DN53185_c1_g3 & 11.67 & $1.31 \mathrm{E}-12$ & Uncharacterized protein ORF91 & \\
\hline TRINITY_DN40074_C2_g1 & 11.21 & 1.49E-08 & Sarcoplasmic calcium-binding protein 1 & \\
\hline TRINITY_DN51715_c1_g6 & 10.96 & $6.10 \mathrm{E}-11$ & Oligopeptide transport ATP-binding protein OppD & OPPD \\
\hline TRINITY_DN46704_C4_g3 & 10.64 & $5.38 \mathrm{E}-07$ & Oligopeptide transport ATP-binding protein OppD & OPPD \\
\hline TRINITY_DN39439_c1_g1 & 10.60 & 4.89E-08 & Myosin heavy chain, muscle & $\mathrm{MHC}$ \\
\hline TRINITY_DN51457_C0_g4 & 10.56 & $9.76 \mathrm{E}-06$ & Arginine kinase & \\
\hline TRINITY_DN44733_C0_g1 & 10.38 & 7.90E-08 & Myosin light chain alkali & MLC1 \\
\hline TRINITY_DN36404_C0_g1 & 10.34 & $5.91 \mathrm{E}-11$ & Collagen alpha-1(III) chain & COL3A1 \\
\hline TRINITY_DN36624_C0_g1 & 10.28 & 8.79E-07 & Myosin regulatory light chain 2 & \\
\hline TRINITY_DN62802_C0_g1 & 10.10 & $2.75 \mathrm{E}-05$ & Ribosomal RNA small subunit methyltransferase $\mathrm{H}$ & $\mathrm{RSMH}$ \\
\hline TRINITY_DN46916_c1_g3 & 9.53 & $2.78 \mathrm{E}-07$ & Tropomyosin & TM1 \\
\hline TRINITY_DN29972_c1_g3 & 9.50 & 4.91E-05 & ATP synthase subunit beta & ATPD \\
\hline TRINITY_DN75810_C0_g1 & 9.44 & $1.72 \mathrm{E}-03$ & DNA-directed RNA polymerase subunit beta' & RPOC \\
\hline TRINITY_DN46916_C1_g2 & 9.26 & $1.53 \mathrm{E}-09$ & Troponin I & \\
\hline TRINITY_DN51457_C0_g2 & 9.13 & $2.81 \mathrm{E}-02$ & Cytochrome c oxidase subunit 1 & $\mathrm{COI}$ \\
\hline TRINITY_DN29188_C0_g1 & 9.09 & 1.09E-06 & Putative transposase InsK for insertion sequence element IS150 & INSK \\
\hline TRINITY_DN30901_C0_g1 & 8.90 & $1.08 \mathrm{E}-04$ & Membrane protein insertase YidC & YIDC \\
\hline TRINITY_DN14841_C0_g1 & 8.88 & $4.80 \mathrm{E}-03$ & L-arabinose transport ATP-binding protein AraG & ARAG \\
\hline TRINITY_DN51715_c1_g8 & 8.76 & $1.02 \mathrm{E}-03$ & Oligopeptide transport ATP-binding protein OppD & OPPD \\
\hline TRINITY_DN55417_c1_g2 & 8.73 & $5.18 \mathrm{E}-03$ & Craniofacial development protein 2 & CFDP2 \\
\hline TRINITY_DN6518_c0_g2 & 8.67 & $6.49 \mathrm{E}-04$ & tRNA-specific 2-thiouridylase MnmA & MNMA \\
\hline TRINITY_DN54848_c1_g1 & 8.58 & $1.22 \mathrm{E}-02$ & Cingulin & CGN \\
\hline TRINITY_DN41914_C0_g2 & 8.55 & $8.58 \mathrm{E}-04$ & von Willebrand factor & WWF \\
\hline TRINITY_DN33939_c0_g1 & 8.53 & $1.43 \mathrm{E}-07$ & Variant surface antigen $C$ & VLPC \\
\hline TRINITY_DN53727_C0_g1 & 8.40 & $2.78 \mathrm{E}-07$ & Troponin T & TNT \\
\hline TRINITY_DN42223_c1_g1 & 8.36 & 4.80E-07 & Actin, alpha cardiac muscle 1 & ACTC1 \\
\hline TRINITY_DN26420_C0_g1 & 8.06 & $1.21 \mathrm{E}-03$ & Fructose-bisphosphate aldolase & ALD \\
\hline TRINITY_DN27809_c1_g2 & 8.00 & $2.00 \mathrm{E}-02$ & 50 S ribosomal protein L6 & RPLF \\
\hline TRINITY_DN50962_c2_g1 & 7.95 & $7.78 \mathrm{E}-03$ & Cytochrome c oxidase subunit 3 & COIII \\
\hline TRINITY_DN50547_c1_g2 & 7.92 & 4.03E-02 & Collagen alpha-2(I) chain & COL1A2 \\
\hline TRINITY_DN29972_C1_g4 & 7.81 & $9.29 \mathrm{E}-03$ & Elongation factor Tu & TUF \\
\hline TRINITY_DN21598_C0_g2 & 7.80 & $2.43 \mathrm{E}-02$ & Chaperone protein DnaK & DNAK \\
\hline TRINITY_DN14984_C0_g1 & 7.79 & $2.54 \mathrm{E}-02$ & Pyruvate dehydrogenase E1 component subunit beta & $\mathrm{PDHB}$ \\
\hline TRINITY_DN4314_CO_g1 & 7.78 & 3.33E-02 & Putative permease MJ0326 & MJ0326 \\
\hline TRINITY_DN71455_C0_g1 & 7.73 & $2.15 \mathrm{E}-04$ & Membrane-associated lipoprotein & UU045 \\
\hline TRINITY_DN27456_C0_g2 & 7.69 & $1.04 \mathrm{E}-03$ & Troponin C, isoform 1 & \\
\hline TRINITY_DN49873_c2_g2 & 7.67 & $2.37 \mathrm{E}-03$ & Cytochrome c oxidase subunit 1 & MT-CO1 \\
\hline TRINITY_DN57252_C0_g1 & 7.65 & $8.30 \mathrm{E}-05$ & Putative ABC transporter ATP-binding protein MG187 homolog & MPN_134 \\
\hline TRINITY_DN76701_C0_g1 & 7.60 & $5.18 \mathrm{E}-03$ & Pyruvate dehydrogenase E1 component subunit beta & $\mathrm{PDHB}$ \\
\hline TRINITY_DN9860_C0_g1 & 7.57 & $2.95 \mathrm{E}-04$ & Ascorbate-specific permease IIC component UlaA & ULAA \\
\hline TRINITY_DN72099_C0_g1 & 7.43 & $1.14 \mathrm{E}-03$ & Lon protease & LON \\
\hline TRINITY_DN37203_c0_g1 & 7.42 & $1.02 \mathrm{E}-03$ & Uncharacterized protein ORF91 & \\
\hline
\end{tabular}


Table 1 Up-regulated differentially expressed genes (DEGs) after refeeding (Continued)

\begin{tabular}{|c|c|c|c|c|}
\hline Gene_ID & $\log 2 \mathrm{FC}$ & padj & Swissprot Description & Symbol \\
\hline TRINITY_DN50867_c4_g2 & 7.39 & $3.41 \mathrm{E}-02$ & Collagen alpha-2( $\mathrm{V})$ chain & COL5A2 \\
\hline TRINITY_DN39233_C1_g2 & 7.17 & $6.64 \mathrm{E}-03$ & Putative uncharacterized transposon-derived protein F52C9.6 & F52C9.6 \\
\hline TRINITY_DN26948_C0_g1 & 7.15 & $6.88 \mathrm{E}-03$ & Troponin C, isotype gamma & \\
\hline TRINITY_DN57481_C0_g1 & 7.12 & $8.41 \mathrm{E}-03$ & $30 S$ ribosomal protein $\mathbf{S} 2$ & RPSB \\
\hline TRINITY_DN42492_C1_g7 & 7.12 & 7.34E-03 & Glyceraldehyde-3-phosphate dehydrogenase & \\
\hline TRINITY_DN76302_C0_g1 & 7.09 & 1.22E-02 & Uncharacterized protein PF11_0207 & PF11_0207 \\
\hline TRINITY_DN20261_CO_g1 & 6.98 & $1.59 \mathrm{E}-02$ & NADH-ubiquinone oxidoreductase chain 2 & MT:ND2 \\
\hline TRINITY_DN31759_C0_g1 & 6.96 & 4.28E-02 & Translation initiation factor IF-2 & INFB \\
\hline TRINITY_DN38000_C0_g1 & 6.92 & $2.90 \mathrm{E}-02$ & RNA-directed DNA polymerase from mobile element jockey & $\mathrm{POL}$ \\
\hline TRINITY_DN66334_C0_g1 & 6.80 & 4.92E-02 & PTS system glucose-specific EllCBA component & PTSG \\
\hline TRINITY_DN50552_CO_g2 & 3.93 & $2.48 \mathrm{E}-04$ & Collagen alpha-2(I) chain & COL1A2 \\
\hline TRINITY_DN41454_C2_g1 & 3.02 & $2.00 \mathrm{E}-02$ & Actin, alpha skeletal muscle & ACTA1 \\
\hline TRINITY_DN39818_C0_g2 & 2.52 & 4.02E-02 & Actin, alpha skeletal muscle 2 & $\mathrm{ACT} 2$ \\
\hline TRINITY_DN37767_C0_g1 & 2.23 & $2.36 \mathrm{E}-02$ & Thymosin beta & TMSB \\
\hline TRINITY_DN48071_CO_g2 & 1.99 & $3.45 \mathrm{E}-03$ & 605 ribosomal protein L22-like 1 & RPL22L1 \\
\hline TRINITY_DN51452_C2_g2 & 1.98 & 7.99E-03 & Troponin T, fast skeletal muscle & TNNT3 \\
\hline TRINITY_DN50109_c0_g2 & 1.94 & 4.60E-02 & Endothelial lipase & LIPG \\
\hline
\end{tabular}

$P$-value $<0.05$ indicated different expression between control and fasting-refeeding groups

The body weight reduction after fasting has been reported in several fish species, including Morone saxatilis [19], Stizostedion vitreum [20], Cynoglossus semilaevis [12], and Oncorhynchus mykiss [21]. Refeeding could accelerate the growth of $S$. hollandi after a period of fasting; this phenomenon is known as compensatory growth and might be affected by feed intake and food utilization efficiency [22, 23]. This has been reported in many fish species, such as Ctenopharyngodon idella [24], Oncorhynchus mykiss [21], Rutilus caspicus [25], and Megalobrama amblycephala [26]. Although starvation and compensatory growth commonly exist in natural and production environments, the molecular mechanisms regulating the growth rates of skeletal muscle under both fasting and refeeding conditions have not been elucidated. The next-generation sequencing technology revolutionized the field of genomics; RNA-Seq can detect the transcriptome of the non-model species without known genomic information [27].

Through comparative transcriptome analysis, most of the up-regulated DEGs under starvation were involved in lipid metabolism, protein degradation and metabolism, and glycometabolism. PNPLA2 is known to be important for triglyceride hydrolysis, and its upregulation might enhance the hydrolysis of triglycerides and produce free fatty acids for other tissues in response to starvation [28]. SLC27A (SLC27A1 and SLC27A3) is associated with fatty acid transport [29], and an increase

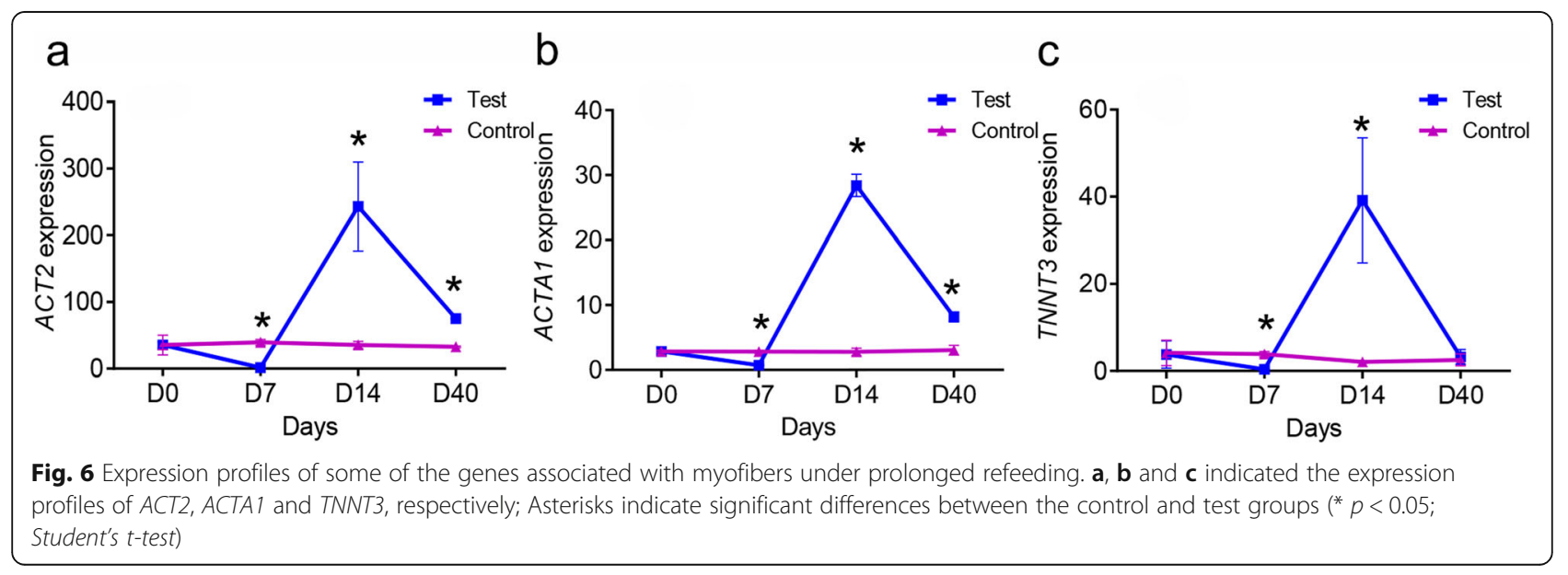



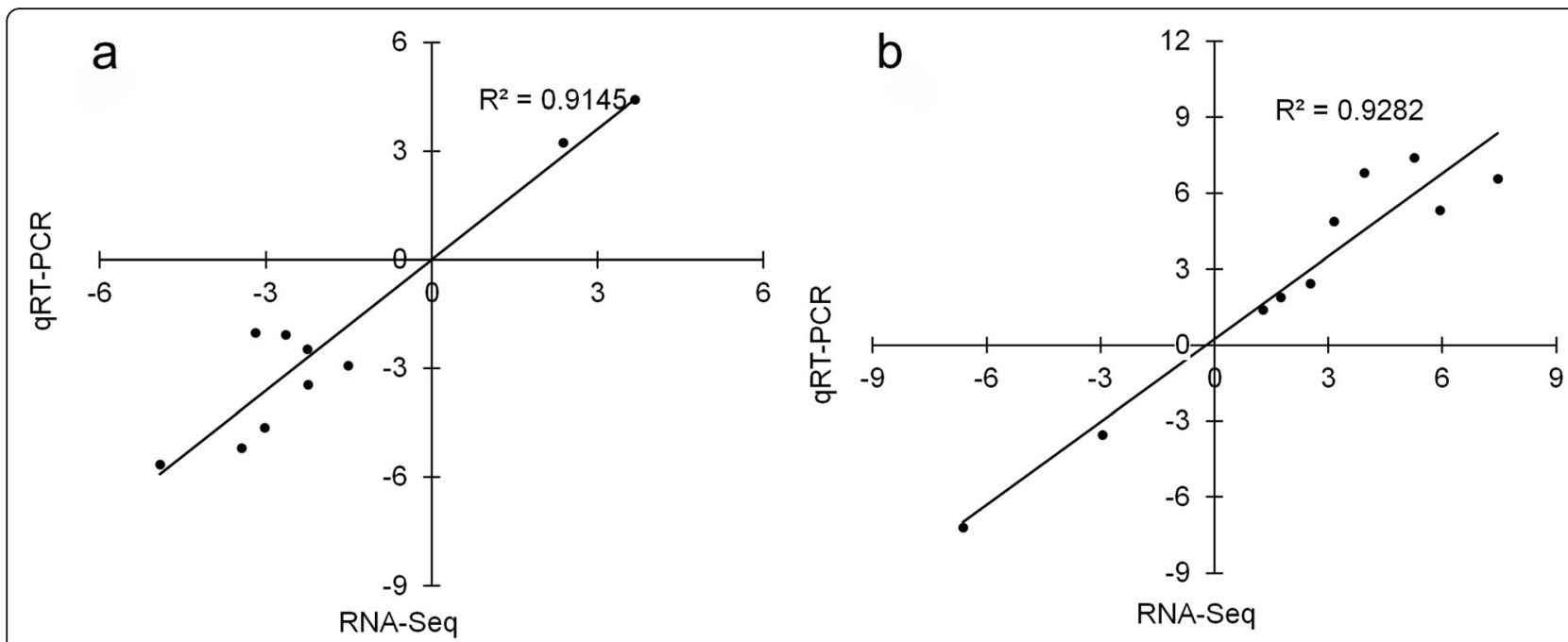

Fig. 7 Correlation between the results of RNA-seq and qRT-PCR. $\mathbf{a}$ and $\mathbf{b}$ represent the correlations between the control and test group (after fasting) and between the control and test group (after refeeding), respectively. The X-axis numbers represent the $\log _{2}$ (fold-change) values from the RNA-seq results. The Y-axis numbers represent the $\log _{2}$ (fold-change) values from the qRT-PCR results

in these genes may contribute to the mobilization of fat. $H A D H$ encodes an enzyme called 3-hydroxyacyl-CoA dehydrogenase, which is important for the betaoxidation of short chain fatty acids in the mitochondria [30]; its increase may contribute to mobilizing the stored fatty acids to provide the necessary energy for the activity of organisms. CPT1s participate in the movement of acylcarnitine from the cytosol into the intermembrane of mitochondria [31], and its up-regulation contributes to fatty acid oxidation. ACACBS (ACACB1 and ACACB2) serve as regulators of mitochondrial fatty acid oxidation through the inhibition of carnitine palmitoyltransferase 1 by its product malonyl-CoA [32], which reduces the fatty acid oxidation rate of organisms. Lipin-1 has phosphatidate phosphatase activity and plays a pivotal role in the maintenance of adipocytes [33]. The up-regulation of these genes may prevent excessive loss of fat during starvation conditions. During fasting, several upregulated DEGs (USP19, RNF25, USP25, USP28, UBE2A, PSMB7, and PSMG3) are involved in the ubiquitinproteasome system, which is one of the major protein degradation systems [34]. In addition, many genes were significantly enriched in amino acid catabolism-related KEGG pathways, including arginine and proline metabolism (ko00330), and valine, leucine, and isoleucine degradation (ko00280). The up-regulation of these genes indicated that fasting could accelerate the consumption of proteins and amino acids. PPP $1 C B$ encodes one of the three catalytic subunits of protein phosphatase 1 , which is a serine/threonine-specific protein phosphatase known to be involved in glycogen metabolism, lipid metabolism, replication, pre-mRNA splicing and protein synthesis [35]. However, the direct connection between this gene and starvation response has not been determined. PFKFB1 is associated with the regulation of the synthesis and degradation of fructose-2,6-biphosphate, which is a rate-limiting enzyme in glucose homeostasis. This protein activates the glycolysis pathway and inhibits the gluconeogenesis pathway to produce more energy for organisms [36], its up-regulation may contribute to the regulation of glucose synthesis and metabolism. GSK3B and GYS are pivotal enzymes in glycogen metabolism $[37,38]$, which may be essential for the response to fasting in S. hollandi. However, the function of the two upregulated genes is opposite, possibly because glycogen may be a buffer of glucose; when excessive stored energy is transformed into carbohydrates, glucose may be transformed into glycogen for temporary storage; when glucose concentration decreases, glycogen may be converted back into glucose. Up-regulated DEGs related to lipid metabolism, protein degradation and metabolism, and glycometabolism suggested that starvation increased the capacity to catabolize fats, proteins, and carbohydrates in S. hollandi. Similar results were found in other fish species: the genes related to fat and carbohydrate catabolism were up-regulated during fasting in large yellow croaker [7], and the genes associated with catabolic pathways and beta oxidation of fatty acids were significantly up-regulated in the muscle of rainbow trout after fasting for 1 month [4].

In addition to the up-regulated DEGs, a total of 912 genes were down-regulated by fasting. Most of these genes were involved in the cell cycle, DNA replication, extracellular matrix, protein synthesis, and immune and stimulus responses. A mitotic cell cycle is a complex process that is regulated by many factors. Cyclin 


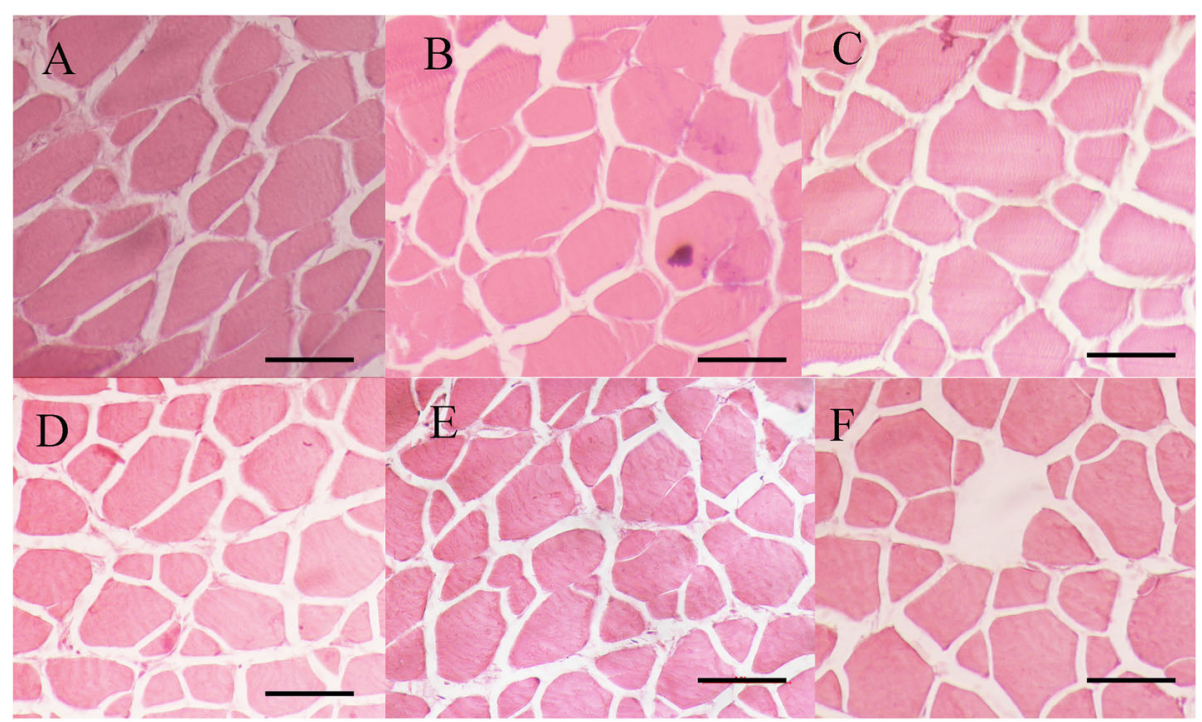

G

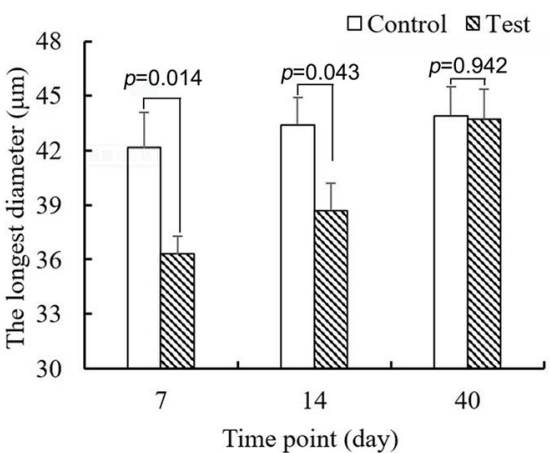

$\mathrm{H}$

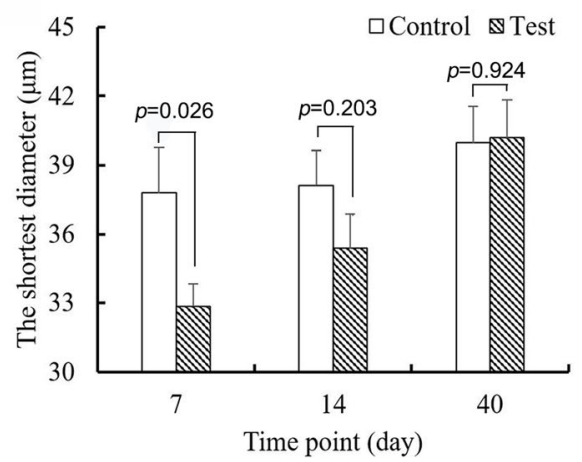

Fig. 8 Changes in myofibers (a-f) indicate myofiber cross-sections in the control group at days 7, 14, 40 and the test group at day 7 (after 7 days fasting), 14 (after 7 days refeeding), 40 (after 33 days refeeding), respectively; $\mathbf{g}$ and $\mathbf{h}$ indicate the longest and shortest diameters of cross-section in the control and test groups, respectively. Asterisks indicate significant differences between the control and test groups $\left(^{*} p<0.05\right.$; Student's $t-$ test; $n=5)$; bars $=50 \mu \mathrm{m}$

(CCNA2 and CCNB1) can regulate cell cycle progress by interacting with cyclin-dependent kinases (CDK1 and CDK2). Wee1 is a nuclear kinase that can prevent cells from undergoing mitosis by inhibiting CDK1; it plays a critical role in the maintenance of normal mitosis during cell division [39, 40]. HRAS is a GTPase involved in the regulation of cell division [41]. F6F is a fibroblast growth factor (FGF) family member that possesses broad mitogenic and cell survival activities and is known to regulate muscle regeneration or differentiation [42]. DNA replication is a vital event in the mitotic cell cycle and showed signs of down-regulation by fasting. A minichromosome maintenance (MCM) complex formed by MCM proteins with DNA helicase activity might be involved in the formation of replication forks and recruitment of other DNA replication-related proteins [43]. PCNA is a cofactor of DNA polymerase delta, which acts as a scaffold in recruiting proteins involved in DNA replication [44]. POLD1 encodes the catalytic subunit of the DNA polymerase delta complex [45], and CDC6 is an essential regulator of DNA replication and plays important roles in the transition from the $S$ phase to the $M$ phase of cell cycle progression [46]. Similar to our findings in S. hollandi, several genes associated with cell cycle and DNA replication were shown to be significantly down-regulated in O. mykiss during fasting [4, 21]. The extracellular matrix comprises a complex mixture of structural and functional macromolecules and is associated with many cellular processes, including growth, differentiation, morphogenesis, survival, and homeostasis [47]. The genes related to the extracellular matrix (ITGA4, COL1A, LAMC1, FN1, TN, and DAG1) were ulated and might reduce the growth and differentiation of myocytes during the fasting period; in addition, several genes associated with the cytoskeleton were down-regulated. Protein synthesis is essential for cellular 
processes. Genes associated with protein synthesis and modification pathways were down-regulated under fasting conditions, which might hinder the growth of S. hollandi. Genes associated with immune and stress tolerance were also down-regulated; heat shock proteins (HSPs) belong to the molecular chaperone family and play important roles in protein folding, as well as in protecting cells from stress. FYN is associated with T-cell signaling [48]; CTSB is a lysosomal cysteine protease associated with immunity [49]. TAP1 and MHCII are related to specific immunity [50] and are important parts of the immune system. The down-regulation of these DEGs suggested that fasting could weaken immunity and might increase morbidity in $S$. hollandi when threatened by pathogenic microorganisms. Previous studies have also shown that many genes related to immune and antimicrobial peptides were down-regulated in the liver following starvation in Atlantic salmon (Salmo salar) [51].

After refeeding, many genes associated with the structure of myofibers were up-regulated. Their expression levels were significantly higher than those in the control group. The sarcomere primarily consists of actin, myosin, tropomyosin, and troponin [52]; the overexpression of these proteins may promote muscle growth [53-55]. Collagens are major components of the perimysium and are essential for the stabilization of the extracellular matrix of muscle [56]. In trout, it was also observed that genes associated with myofiber and muscle remodeling were up-regulated 7 to 36 days post-refeeding [4], and the same research team suggested that compensatory muscle growth response resulted from the stimulation of hypertrophy [21]. Furthermore, several genes associated with energy metabolism (ATPD, GAPDH, ND2, and $P D H B$ ) were up-regulated; they might be required for meeting the energy requirement for muscle growth. In general, these findings indicated that the accelerated biosynthesis of myofibers resulted in compensatory growth following refeeding in S. hollandi. In histology analysis, the significant difference in the dimensions of myofibers showed that starvation can inhibit the growth of myofibers, which was in accord with results obtained from transcriptome analysis. After refeeding, the overgrowth of myofibers was consistent with the up-regulated expression levels of genes associated with muscle structure. Based on the above results, we suggest that muscle overgrowth may play an important role in the compensatory growth of fish.

\section{Conclusions}

In the present experiment, we carried out an analysis of the transcriptome profiles of $S$. hollandi muscle under different breeding conditions. During fasting, genes involved in fat, protein, and carbohydrate metabolism were up-regulated, which indicated that the stored energy was mobilized. On the other hand, the expression levels of genes associated with the cell cycle, DNA replication, and cellular structures decreased, which suggested that growth might be inhibited by starvation. After refeeding, most of the genes down-regulated during fasting were recovered, while many genes associated with the structure of myofibers were up-regulated. Histological analyses of muscle also showed muscle overgrowth after refeeding. The results indicated that muscle hypertrophy may play an important role in compensatory growth after fasting refeeding. The results of these experiments may help to elucidate the mechanisms underlying the starvation response and compensatory growth.

\section{Methods}

\section{Sample preparation and fasting experiments}

The mixed-sex, full-sib experimental fish were obtained from Shaoguan Fisheries Research Institute in Guangdong, China. A total of 210 fish with an approximate weight of $1.0 \mathrm{~g}$ were randomly and equally assigned to seven aquariums. As Spinibarbus hollandi is highly sensitive to the external stimulus, which will lead to not ingesting for several days, one aquarium was sampled only once in our experiment. Thus, among the seven aquariums, one was used for the measurements of the initial growth traits, and the remaining six aquariums were equally divided into test and control groups, corresponding to 7,14 , and 40 days. To minimize the effects introduced by different aquariums, we maintained all aquariums in the same conditions, including $100 \mathrm{~L}$ of charcoal-dechlorinated and continuous flow-through tap water $(\mathrm{pH} 7.0 \pm 0.1)$ with forced-air aeration, and the temperature of the water was $26^{\circ} \mathrm{C}$ with a photoperiod of 14:10 h (light:dark). The fish were manually fed twice a day to apparent satiation at 7:00 and 17:00 h, with a $35 \%$ protein $/ 4 \%$ fat commercial diet (QiCai Pet Products Co., Ltd., Guangzhou, China) for 2 weeks. Fish served as control groups and were fed to satiation every day as mentioned above. For the test groups, fish were first deprived of food for 7 days and then fed twice per day for 33 days. For aquariums used for 7-day continuously feeding, 7-day fasting and 7-day refeeding after fasting, 15 fish were used for RNA-seq, 3 fish were used for qRT-PCR, 5 fish were used for histological analysis, and redundant 7 fish were used for prevent accidental death. For other aquariums, 3 fish were used to qRT-PCR, 5 fish were used to histological analysis, redundant fish were used to keep same breeding density in all aquariums and prevent accidental death. Eight fish were randomly selected for measure of growth traits in each aquarium. After experiments, the remained fish were bred continuously for further studies. Both groups were sampled sequentially on days $0,7,14$, and 40 . The fish were put into water with an overdose $(100 \mathrm{ppm})$ of 
eugenol (Sangon Biotech Co., Ltd., Shanghai, China) for $10 \mathrm{~min}$ to anesthetize them before dissection, and their body weights, body lengths, total lengths, body thicknesses and body heights were measured. Daily gain of body weight was also calculated using the following formula:

$$
\mathrm{R}=(\mathrm{m} 2-\mathrm{m} 1) / \mathrm{t}
$$

The $\mathrm{R}$ indicated the daily body weight gain ((g/day), the $\mathrm{m} 1$ indicated the initial weight $(\mathrm{g})$, the $\mathrm{m} 2$ indicated the final weight after treatment $(\mathrm{g})$, and $\mathrm{t}$ indicated the period of treatment (day).

For RNA sequencing, The muscle tissues were rapidly sampled from the same location of dorsal white muscle under dorsal fin from all fish without considering dorsal side, immediately frozen in liquid nitrogen, and stored at $-80^{\circ} \mathrm{C}$ for further testing. At the same time, another three and five fish were sampled as the above methods for qRT-PCR and histology analysis, respectively. After the experiment, wherever possible, most remaining experimental fish were retained for other research.

\section{RNA isolation}

Total RNA was extracted from approximately $80 \mathrm{mg}$ of muscle tissue using RNAiso reagents (Takara, Dalian, China) according to the manufacturer's instructions. The quantity and quality of RNA samples were determined using a microplate spectrophotometer (BioTek Company, USA) followed by electrophoresis on a $1 \%$ agarose gel. The RNA samples were stored at $-80^{\circ} \mathrm{C}$ until further use.

\section{Library preparation and sequencing}

The RNA samples prepared above were used for RNAseq. The variation among individuals was minimized by mixing equal amounts of RNA from five samples in the same group to generate three replicated mixed RNA pools. A total of nine replicated mixed RNA samples were used for cDNA library preparation. RNA-seq libraries were prepared using NEBNext UltraTM RNA Library Prep Kit (NEB, USA) following the manufacturer's protocols. Briefly, mRNA was purified from total RNA and was broken to fragmentations using divalent cations. First strand cDNA was synthesized using reverse transcriptase (MMLV), and second strand cDNA was synthesized using DNA polymerase $\mathrm{I}$, and the remained mRNA were subsequently removed using RNase $\mathrm{H}$. cDNA fragments were ligated with adaptor and then 250 300 bp fragments were selected using AMPure beads for library construction (Beckman Coulter, Beverly, USA). $3 \mu$ l USER Enzyme (NEB, USA) was added into purified cDNA fragments and incubated at $37^{\circ} \mathrm{C}$ for $15 \mathrm{~min}$ followed by $5 \mathrm{~min}$ at $95^{\circ} \mathrm{C}$. cDNA fragments were amplified using polymerase chain reaction (PCR). After purified by using AMPure beads, the library quality and quantity was measured using Agilent 2100 Bioanalyzer (Agilent Technologies). Finally, the libraries were sent to Novogene Co. Ltd. to sequence using IlluminaHiseq 2000 platform with PE-150 paired-end approach.

\section{RNA-seq data analyses}

Raw data were filtered by removing the reads containing the adapters, poly- $\mathrm{N}$, and low-quality reads, resulting in the generation of clean reads, which were assembled de novo using the Trinity software version 2.5.1 [57]. The assembled transcripts were identified using Blast+ (version: ncbi-blast2.2.28+) against the National Center for Biotechnology Information (NCBI) nonredundant (Nr, e-value $<1 \mathrm{e}-5)$, Clusters of Orthologous Groups of proteins (COG, e-value $<1 \mathrm{e}^{-3}$ ), Kyoto Encyclopedia of Genes and Genomes (KEGG, e-value <1e-5), and Swiss-Prot databases (e-value < 1e-5). The gene ontology (GO) annotation was performed using Blast2GO with an e-value threshold of 1e-6.

The total mapped read counts for each transcript were determined using the RSEM software v1.2.15 [58]. The differential expression statistical analyses were performed using the median of ratios from the $\mathrm{R}$ package, DESeq [59]. The adjusted $p$-value (p-adj) with a cut-off value of 0.05 and $\log 2$ (fold change) with a cut-off absolute value of 1 were used to identify the significant differentially expressed genes (DEGs). Omicshare online website (http://www.omicshare.com/tools/Home/Soft/ pathwaygsea?l=en-us) was used for Gene Ontology and KEGG pathway enrichment analysis. Significantly enriched pathways in the given gene set compared to the genome background are defined by a hypergeometric test. The formula for calculating the $P$-value is

$$
P=1-\sum_{i=0}^{m-1} \frac{\left(\begin{array}{c}
M \\
i
\end{array}\right)\left(\begin{array}{c}
N-M \\
n-i
\end{array}\right)}{\left(\begin{array}{c}
N \\
n
\end{array}\right)}
$$

In this equation, $\mathrm{N}$ is the number of all genes with KEGG pathway annotation, $\mathrm{n}$ is the gene number of given genes set in $\mathrm{N}, \mathrm{M}$ is the number of all genes that are annotated to the certain pathway, and $\mathrm{m}$ is the gene number in the given gene set that is annotated to the certain pathways. The calculated p-value is gone through FDR Correction, taking FDR $\leq 0.05$ as a threshold. GO terms with a q-value lower than 0.05 were further summarized with REVIGO [60]. The UpSet Chart were made by an R package UpSetR [61].

\section{Quantitative real-time PCR analysis}

Three biological replicates were generated for each RNA sample for quantitative real-time PCR (qRT-PCR) 
analysis. The beta-actin gene was used as an internal control, which did not significantly change expression in the experiment (Control vs Fasting, $p=0.80$; Control vs Refeeding, $p=0.82$; Fasting vs Refeeding, $p=1.0$; exactTest, DESeq). The primers used in the qRT-PCR analysis are shown in Additional file 1: Table S1. The qRT-PCR analysis was performed using the ABI 7000 platform in $20 \mu \mathrm{L}$ reactions containing the following components: $100 \mathrm{ng}$ of cDNA, $10 \mu \mathrm{L}$ Power SYBR Green PCR Master Mix (Vazyme Biotech, Nanjing, China), $0.3 \mu \mathrm{L}$ of each primer $(10 \mu \mathrm{M})$, and $7.4 \mu \mathrm{L}$ double-distilled water. The reaction procedure was as mentioned in the manufacturer's instructions for the SYBR Green PCR Master Mix. All samples were analyzed in triplicate, and fold changes were calculated using the comparative $\mathrm{Ct}$ method (also known as the $2^{-\Delta \Delta C t}$ method). All data are provided in terms of the relative mRNA expression as the mean \pm S.E. $(n=3)$.

\section{Muscle histology}

For muscle morphology observation, five fish from each group were selected. The dorsal muscle tissues obtained in the preceding experiment were fixed for $24 \mathrm{~h}$ in Bouin solution. The tissues were then dehydrated using an ethyl alcohol series, hyalinized in xylene baths, and embedded in paraffin. After the paraffin block solidified, thin sections $(6 \mu \mathrm{m})$ were cut using a rotary microtome (Cut 4055; Olympus American, Melville, NY, USA). The sections were flattened, stained with hematoxylin and eosin, mounted on slides with neutral resin, and examined using light microscopy. The longest and shortest diameters of myofibers were measured and recorded for the control and test groups. A total of $\sim 100$ cells of each group were randomly selected for measurement. For each fish, a clear vision was used to measure cell size, and approximately 20 cells of each vision were randomly measured and recorded.

\section{Statistical analyses}

All data were analyzed using SPSS Statistics software version 20 (IBM, Chicago, IL, USA) and presented as the means \pm SEM. The body weight and myocyte sizes of fish in the control and test groups were analyzed using Student's $t$-test. $P$-values $<0.05$ were considered to be statistically significant.

\section{Supplementary information}

Supplementary information accompanies this paper at https://doi.org/10. 1186/s12864-019-6345-2.

Additional file 1: Table S1. Primer pairs used for GRT-PCR. Figure S1. Daily changes of growth traits. Figure S2. The expression of genes associated with structure of myofiber.
Additional file 2: Table S2. Figure S2. The list of all differentially expressed genes during fasting-refeeding.

\section{Abbreviations}

COG: Clusters of Orthologous Groups of proteins; DEGs: Differentially expressed genes; FPKM: Fragment Per Kilobase of transcript sequence per Millions; GO: Gene ontology; HSPs: Heat shock proteins; KEGG: Kyoto Encyclopedia of Genes and Genomes; MCM: Mini-chromosome maintenance; NCBI: National Center for Biotechnology Information (); Nr: Non-redundant protein (NR), NCBI database; Nt: Non-redundant nucleotide (Nt), NCBI database; qRT-PCR: Quantitative real-time PCR

\section{Acknowledgments}

We thank the anonymous reviewers for their helpful comments on this work.

\section{Authors' contributions}

HS designed the experiment, HQZ and LPH obtained permissions to collect the samples and measure the growth traits. YY isolated the RNA, YY and KX analyzed the data. The manuscripts were written and revised by $Y Y, H S, H Q Z$, $\mathrm{LPH}$ and KX. All authors have read and approved the final manuscript.

\section{Funding}

This work was financially supported by Ministry of Agriculture and Rural Affairs of the People's Republic of China (No.201303048) and Department of Science and Technology of Guangdong Province (Nos. 2014A020208145).

The funders had no role in the designing and conducting of this study and collection, analysis, and interpretation of data and in writing the manuscript.

\section{Availability of data and materials}

All the data obtained in the current study have been presented in this article. The RNA-Seq sequence raw data-set supporting the results of this study have been deposited at the National Center for Biotechnology Information (NCBI), sequence read archive (SRA) database, and the accession number is SRP133262.

\section{Ethics approval and consent to participate}

This study was approved by the Animal Care and Use committee in the School of Life Science at Guangzhou University.

Consent for publication

Not applicable.

\section{Competing interests}

The authors declare that they have no competing interests.

Received: 21 April 2019 Accepted: 27 November 2019

Published online: 05 December 2019

References

1. Benzekri H, Armesto P, Cousin X, Rovira M, Crespo D, Merlo MA, Mazurais D, Bautista R, Guerrero-Fernández D, Fernandez-Pozo N, et al. De novo assembly, characterization and functional annotation of Senegalese sole (Solea senegalensis) and common sole (Solea solea) transcriptomes: Integration in a database and design of a microarray. BMC Genomics. 2014; 15(1):952. https://doi.org/10.1186/1471-2164-15-952.

2. Ma D, Ma A, Huang Z, Wang G, Wang T, Xia D, Ma B. Transcriptome analysis for identification of genes related to gonad differentiation, growth, immune response and marker discovery in the turbot (Scophthalmus maximus). PLoS ONE. 2016;11(2):e0149414. https://doi.org/10.1371/journal.pone.0149414.

3. Takahashi LS, Biller JD, Criscuolo-Urbinati E, Urbinati EC. Feeding strategy with alternate fasting and refeeding: effects on farmed pacu production. J Anim Physiol Anim Nutr. 2011:95(2):259-66. https://doi.org/10.1111/j.14390396.2010.01050.x

4. Rescan PY, Montfort J, Rallière C, Le Cam A, Esquerré D, Hugot K. Dynamic gene expression in fish muscle during recovery growth induced by a fasting-refeeding schedule. BMC Genomics. 2007:8:438. https://doi.org/10. 1186/1471-2164-8-438

5. Keogh K, Kenny DA, Cormican P, Kelly AK, Waters SM. Effect of dietary restriction and subsequent re-alimentation on the transcriptional profile of 
hepatic tissue in cattle. BMC Genomics. 2016;17(1):244. https://doi.org/10. 1186/s12864-016-2578-5.

6. Liu L, Li C, Su B, Beck BH, Peatman E. Short-Term Feed Deprivation Alters Immune Status of Surface Mucosa in Channel Catfish (Ictalurus punctatus). PLoS ONE. 2013;8(9):e74581. https://doi.org/10.1371/journal.pone.0074581.

7. Qian B, Xue L, Huang H. Liver transcriptome analysis of the large yellow croaker (Larimichthys crocea) during fasting by using RNA-seq. PLoS ONE. 2016;11(3):e0150240. https://doi.org/10.1371/journal.pone.0150240.

8. Hornick JL, Van Eenaeme C, Gérard O, Dufrasne I, Istasse L. Mechanisms of reduced and compensatory growth. Domest Anim Endocrinol. 2000;19(2): 121-32. https://doi.org/10.1016/S0739-7240(00)00072-2.

9. Johansen SJS, Ekli M, Stangnes B, Jobling M. Weight gain and lipid deposition in Atlantic salmon, Salmo salar, during compensatory growth: evidence for lipostatic regulation? Aquac Res. 2001;32(12):963-74. https:// doi.org/10.1046/j.1365-2109.2001.00632.x.

10. Tian X, Qin JG. A single phase of food deprivation provoked compensatory growth in barramundi Lates calcarifer. Aquaculture. 2003;224(1-4):169-79. https://doi.org/10.1016/50044-8486(03)00224-2.

11. Reigh RC, Williams MB, Jacob BJ. Influence of repetitive periods of fasting and satiation feeding on growth and production characteristics of channel catfish, Ictalurus punctatus. Aquaculture. 2006;254(1-4):506-16. https://doi. org/10.1016/j.aquaculture.2005.10.043.

12. Tian X, Fang J, Dong S. Effects of starvation and recovery on the growth, metabolism and energy budget of juvenile tongue sole (Cynoglossus semilaevis). Aquaculture. 2010;310(1-2):122-9. https://doi.org/10.1016/j. aquaculture.2010.10.021

13. Russell NR, Wootton RJ. Appetite and growth compensation in the European minnow, Phoxinus phoxinus (Cyprinidae), following short periods of food restriction. Environ Biol Fish. 1992;34(3):277-85. https://doi.org/10. 1007/BF00004774.

14. Xie S, Zhu X, Cui Y, Wootton RJ, Lei W, Yang Y. Compensatory growth in the gibel carp following feed deprivation: temporal patterns in growth, nutrient deposition, feed intake and body composition. J Fish Biol. 2001; 58(4):999-1009. https://doi.org/10.1006/fbi.2000.1505.

15. Loughna PT, Goldspink $G$. The effects of starvation upon protein turnover in red and white myotomal muscle of rainbow trout, Salmo gairdneri Richardson. J Fish Biol. 1984;25(2):223-30. https://doi.org/10.1111/j.1095-8649.1984.tb04869.x.

16. Weatherley AH, Gill HS. Growth dynamics of white myotomal muscle fibres in the bluntnose minnow, Pimephales notatus Rafinesque, and comparison with rainbow trout, Salmo gairdneri Richardson. J Fish Biol. 1984;25(1):13-24. https://doi.org/10.1111/j.1095-8649.1984.tb04846.X.

17. Yang Y, Lan Z, Shu H, Zhou H, Jiang X, Hou L, Gu P. Association between expression levels and growth trait-related SNPs located in promoters of the MC4R and MSTN genes in Spinibarbus hollandi. Genes Genomics. 2018; 40(11):1119-25. https://doi.org/10.1007/s13258-018-0666-4.

18. Yang Y, Li Q, Shu H, Zhou H, Li X, Hou L. Characterization of the melanocortin-4 receptor gene from Spinibarbus hollandi and the association between its polymorphisms and S. hollandi growth traits. Fish Sci. 2017; 83(6):967-76. https://doi.org/10.1007/s12562-017-1125-x.

19. Small BC, Soares JH, Woods LC, Dahl GE. Communications effect of fasting on pituitary growth hormone expression and circulating growth hormone levels in striped bass. N Am J Aquac. 2002;64(4):278-83. https://doi.org/10. 1577/1548-8454(2002)064<0278:EOFOPG>2.0.CO;2.

20. Czesny S, Rinchard J, Abiado MAG, Dabrowski K. The effect of fasting, prolonged swimming, and predator presence on energy utilization and stress in juvenile walleye (Stizostedion vitreum). Physiol Behav. 2003;79(4-5): 597-603. https://doi.org/10.1016/50031-9384(03)00124-0.

21. Rescan PY, Cam A, Rallière C, Montfort J. Global gene expression in muscle from fasted/refed trout reveals up-regulation of genes promoting myofibre hypertrophy but not myofibre production. BMC Genomics. 2017;18(1):447. https://doi.org/10.1186/s1 2864-017-3837-9.

22. Ali M, Nicieza A, Wootton RJ. Compensatory growth in fishes: a response to growth depression. Fish Fish. 2003;4(2):147-90. https://doi.org/10.1046/j. 1467-2979.2003.00120.x.

23. Jobling M, Johansen SJS. The lipostat, hyperphagia and catch-up growth. Aquac Res. 1999;30(7):473-8. https://doi.org/10.1046/.1365-2109.1999.00358.X

24. He L, Pei Y, Jiang Y, Li Y, Liao L, Zhu Z, Wang Y. Global gene expression patterns of grass carp following compensatory growth. BMC Genomics. 2015;16(1):184. https://doi.org/10.1186/s12864-015-1427-2.

25. Abolfathi M, Hajimoradloo A, Ghorbani R, Zamani A. Compensatory growth in juvenile roach Rutilus caspicus: effect of starvation and re-feeding on growth and digestive surface area. J Fish Biol. 2012;81(6):1880-90. https:// doi.org/10.1111/j.1095-8649.2012.03407.x.

26. Zhu K, Chen L, Zhao J, Wang H, Wang W, Li Z, Wang H. Molecular characterization and expression patterns of myogenin in compensatory growth of Megalobrama amblycephala. Comp Biochem Physiol B Biochem Mol Biol. 2014;170(1):10-7. https://doi.org/10.1016/j.cbpb.2014.01.001.

27. Qian X, Ba Y, Zhuang Q, Zhong G. RNA-seq technology and its application in fish transcriptomics. OMICS. 2014;18(2):98-110. https://doi.org/10.1089/ omi.2013.0110.

28. Jenkins CM, Mancuso DJ, Yan W, Sims HF, Gibson B, Gross RW. Identification, cloning, expression, and purification of three novel human calcium-independent phospholipase $\mathrm{A}<\mathrm{inf}>2</$ inf $>$ family members possessing triacylglycerol lipase and acylglycerol transacylase activities. J Biolog Chem. 2004;279(47):48968-75. https://doi.org/10.1074/jbc. M407841200.

29. Kowalewska-Łuczak I, Czerniawska-Piątkowska E, Pecka-Kiełb E. Investigation on relationships of the FABP3 and SLC27A3 genes with milk production traits in sheep. J Elem. 2017;22(4):1485-93. https://doi.org/10.5601/jelem.2017.22.1.1406.

30. Barycki JJ, O'Brien LK, Strauss AW, Banaszak LJ. Sequestration of the active site by interdomain shifting: Crystallographic and spectroscopic evidence for distinct conformations of L-3-hydroxyacyl-CoA dehydrogenase. J Biol Chem. 2000;275(35):27186-96. https://doi.org/10.1074/jbc.M004669200.

31. Bonnefont JP, Djouadi F, Prip-Buus C, Gobin S, Munnich A, Bastin J. Carnitine palmitoyltransferases 1 and 2: biochemical, molecular and medical aspects. Mol Asp Med. 2004;25(5-6):495-520. https://doi.org/10.1016/j.mam. 2004.06.004

32. Lee CK, Cheong HK, Ryu KS, Jae IL, Lee W, Young HJ, Cheong C. Biotinoyl domain of human acetyl-CoA carboxylase: structural insights into the carboxyl transfer mechanism. Proteins Struct Funct Genet. 2008;72(2):61324. https://doi.org/10.1002/prot.21952.

33. Ren H, Federico L, Huang H, Sunkara M, Drennan T, Frohman MA, Smyth SS, Morris AJ. A phosphatidic acid binding/nuclear localization motif determines lipin1 function in lipid metabolism and adipogenesis. Mol Biol Cell. 2010;21(18):3171-81. https://doi.org/10.1091/mbc.E10-01-0073.

34. Guharoy M, Bhowmick P, Sallam M, Tompa P. Tripartite degrons confer diversity and specificity on regulated protein degradation in the ubiquitinproteasome system. Nat Commun. 2016;7:10239. https://doi.org/10.1038/ ncomms10239.

35. Lee JH, You J, Dobrota E, Skalnik DG. Identification and characterization of a novel human PP1 phosphatase complex. J Biol Chem. 2010;285(32):2446676. https://doi.org/10.1074/jbc.M1 10.109801.

36. Pilkis SJ, Elmaghrabi MR, Claus TH. Fructose-2,6-bisphosphate in control of hepatic gluconeogenesis. From metabolites to molecular genetics. Diabetes Care. 1990;13(6):582-99.

37. Embi N, Rylatt DB, Cohen P. Glycogen synthase Kinase-3 from rabbit skeletal muscle: separation from cyclic-AMP-dependent protein kinase and Phosphorylase kinase. Eur J Biochem. 1980;107(2):519-27. https://doi.org/10. 1111/j.1432-1033.1980.tb06059.x.

38. Jentjens $R$, Jeukendrup AE. Determinants of post-exercise glycogen synthesis during short-term recovery. Sports Med. 2003;33(2):117-44. https:// doi.org/10.2165/00007256-200333020-00004.

39. Den Haese GJ, Walworth N, Carr AM, Gould KL. The Wee1 protein kinase regulates T14 phosphorylation of fission yeast Cdc2. Mol Biol Cell. 1995;6(4): 371-85. https://doi.org/10.1091/mbc.6.4.371.

40. Garnier D, Loyer P, Ribault C, Guguen-Guillouzo C, Corlu A. Cyclindependent kinase 1 plays a critical role in DNA replication control during rat liver regeneration. Hepatology. 2009;50(6):1946-56. https://doi.org/10. 1002/hep.23225.

41. Guil S, De La Iglesia N, Fernández-Larrea J, Cifuentes D, Ferrer JC, Guinovart $\mathrm{JJ}$, Bach-Elias M. Alternative splicing of the human proto-oncogene $\mathrm{C}-\mathrm{H}$-ras renders a new Ras family protein that trafficks to cytoplasm and nucleus. Cancer Res. 2003;63(17):5178-87.

42. Armand AS, Launay T, Pariset C, Della Gaspera B, Charbonnier F, Chanoine C. Injection of FGF6 accelerates regeneration of the soleus muscle in adult mice. Biochim Biophys Acta. 2003;1642(1-2):97-105. https://doi.org/10.1016/ S0167-4889(03)00103-4.

43. Maiorano D, Lemaitre JM, Méchali M. Stepwise regulated chromatin assembly of MCM2-7 proteins. J Biol Chem. 2000;275(12):8426-31. https:// doi.org/10.1074/jbc.275.12.8426.

44. Moldovan GL, Pfander B, Jentsch S. PCNA, the maestro of the replication fork. Cell. 2007;129(4):665-79. https://doi.org/10.1016/j.cell.2007.05.003. 
45. Prindle MJ, Loeb LA. DNA polymerase delta in dna replication and genome maintenance. Environ Mol Mutagen. 2012;53(9):666-82. https://doi.org/10. 1002/em.21745.

46. Liu J, Smith CL, DeRyckere D, DeAngelis K, Martin GS, Berger JM. Structure and function of $\mathrm{Cdc} 6 / \mathrm{Cdc} 18$ : implications for origin recognition and checkpoint control. Mol Cell. 2000;6(3):637-48. https:/ doi.org/10.1016/S1097-2765(00)00062-9.

47. Theocharis AD, Skandalis SS, Gialeli C, Karamanos NK. Extracellular matrix structure. Adv Drug Deliv Rev. 2016;97:4-27. https://doi.org/10. 1016/j.addr.2015.11.001.

48. Palacios EH, Weiss A. Function of the Src-family kinases, Lck and Fyn, in Tcell development and activation. Oncogene. 2004;23(48 REV. ISS. 7):79908000. https://doi.org/10.1038/sj.onc.1208074.

49. Rasid O, Mériaux V, Khan EM, Borde C, Ciulean IS, Fitting C, Manoury B, Cavaillon JM, Doyen N. Cathepsin B-Deficient Mice Resolve Leishmania major Inflammation Faster in a T Cell-Dependent Manner. PLoS Neglected Tropical Diseases. 2016;10(5):e0004716. https://doi.org/10. 1371/journal.pntd.0004716.

50. Mambres DH, MacHelart A, Potemberg G, De Trez C, Ryffel B, Letesson JJ, Muraille $E$. Identification of immune effectors essential to the control of primary and secondary intranasal infection with brucella melitensis in mice. J Immunol. 2016;196(9):3780-93. https://doi.org/10.4049/jimmunol.1502265.

51. Martin SAM, Douglas A, Houlihan DF, Secombes CJ. Starvation alters the liver transcriptome of the innate immune response in Atlantic salmon (Salmo salar). BMC Genomics. 2010;11(1):418. https://doi.org/10.1186/ 1471-2164-11-418

52. Ono S. Dynamic regulation of sarcomeric actin filaments in striated muscle. Cytoskeleton. 2010;67(11):677-92. https://doi.org/10.1002/cm.20476.

53. Sanger JW, Wang J, Fan Y, White J, Mi-Mi L, Dube DK, Sanger JM, Pruyne D. Assembly and maintenance of myofibrils in striated muscle. Handb Exp Pharmacol. 2017;235:39-75. https://doi.org/10.1007/164_2016_53.

54. Gabriel Kuniyoshi ML, Nunes Da Silva-Gomes R, Cavalcante Souza Vieira J, Casemiro Hessel M, Assunção Mareco E, Dos Santos VB, Carvalho RF, De Magalhães Padilha P, Dal-Pai-Silva M. Proteomic analysis of the fast-twitch muscle of pacu (Piaractus mesopotamicus) after prolonged fasting and compensatory growth. Comp Biochem Physio Part D Genomics Proteomics. 2019:30:321-32. https://doi.org/10.1016/i.cbd.2019.04.005

55. Wang J, Ge Q, Li J, Chen Z, Li J. Isolation and characterization of three skeletal troponin genes and association with growth-related traits in Exopalaemon carinicauda. Mol Biol Rep. 2019;46(1):705-18. https://doi.org/ 10.1007/s11033-018-4526-8.

56. McCormick RJ. The flexibility of the collagen compartment of muscle. Meat Sci. 1994;36(1-2):79-91. https://doi.org/10.1016/0309-1740(94)90035-3.

57. Grabherr MG, Haas BJ, Yassour M, Levin JZ, Thompson DA, Amit I, Adiconis X, Fan L, Raychowdhury R, Zeng Q, et al. Full-length transcriptome assembly from RNA-Seq data without a reference genome. Nat Biotechnol. 2011;29(7): 644-52. https://doi.org/10.1038/nbt.1883.

58. Li B, Dewey CN. RSEM: accurate transcript quantification from RNA-sea data with or without a reference genome. In: Bioinformatics: The Impact of Accurate Quantification on Proteomic and Genetic Analysis and Research; 2014. p. 41-74.

59. Anders $\mathrm{S}$, Huber W. Differential expression analysis for sequence count data. Genome Biol. 2010;11(10):R106. https://doi.org/10.1186/gb-2010-11-10-r106.

60. Supek F, Bosnjak M, Skunca N, Smuc T. REVIGO summarizes and visualizes long lists of gene ontology terms. PLoS One. 2011;6:e21800.

61. Conway J, Lex A, Gehlenborg N. UpSetR: an R package for the visualization of intersecting sets and their properties. Bioinformatics. 2017;33(18):2938-40.

\section{Publisher's Note}

Springer Nature remains neutral with regard to jurisdictional claims in published maps and institutional affiliations.

\section{Ready to submit your research? Choose BMC and benefit from:}

- fast, convenient online submission

- thorough peer review by experienced researchers in your field

- rapid publication on acceptance

- support for research data, including large and complex data types

- gold Open Access which fosters wider collaboration and increased citations

- maximum visibility for your research: over $100 \mathrm{M}$ website views per year

At BMC, research is always in progress.

Learn more biomedcentral.com/submissions 